\title{
A Fast Algorithm for Augmenting Edge-Connectivity by One with Bipartition Constraints
}

\author{
Tadachika OKI $^{\dagger a}$, Student Member, Satoshi TAOKA ${ }^{\dagger}$, Toshiya MASHIMA ${ }^{\dagger \dagger}$, Members, \\ and Toshimasa WATANABE ${ }^{\dagger}$, Fellow
}

\begin{abstract}
SUMMARY The $k$-edge-connectivity augmentation problem with bipartition constraints ( $k \mathrm{ECABP}$, for short) is defined by "Given an undirected graph $G=(V, E)$ and a bipartition $\pi=\left\{V_{B}, V_{W}\right\}$ of $V$ with $V_{B} \cap V_{W}=$ $\emptyset$, find an edge set $E_{f}$ of minimum cardinality, consisting of edges that connect $V_{B}$ and $V_{W}$, such that $G^{\prime}=\left(V, E \cup E_{f}\right)$ is $k$-edge-connected." The problem has applications for security of statistical data stored in a cross tabulated table, and so on. In this paper we propose a fast algorithm for finding an optimal solution to $(\sigma+1) \mathrm{ECABP}$ in $O\left(|V||E|+\left|V^{2}\right| \log |V|\right)$ time when $G$ is $\sigma$-edge-connected $(\sigma>0)$, and show that the problem can be solved in linear time if $\sigma \in\{1,2\}$.

key words: connectivity augmentation problems, edge-connectivity, bipartition constraints, graphs, polynomial time algorithms
\end{abstract}

\section{Introduction}

[Background] The k-edge-connectivity augmentation problem ( $k \mathrm{ECA}$, for short) is defined by "Given a multigraph $G=(V, E)$, find an edge set $E_{f}$ of minimum cardinality such that $G^{\prime}=\left(V, E \cup E_{f}\right)$ is $k$-edge-connected, where a multigraph means a graph, with unweighted edges, such that multiple edges may exist." We often denote $G^{\prime}$ as $G+E_{f}$, and $E_{f}$ is called an optimal solution to the problem. There are several applications for construction of a fault-tolerant network, and so on. It is called the $k$-edge-connectivity augmentation problem with bipartition constraints ( $k \mathrm{ECABP}$, for short) when a bipartition $\pi=\left\{V_{B}, V_{W}\right\}$ of $V$ with $V_{B} \cap V_{W}=\emptyset$ is additionally given and we require that $E_{f}$ consists of edges connecting between $V_{B}$ and $V_{W}$ (see Fig. 1).

A bipartite graph is a graph $(V, E)$ such that $V$ is partitioned into two sets $V^{B}$ and $V^{W}$, and any edge $(u, v) \in E$ satisfies a condition $\left(u \in V^{B}\right.$ and $\left.v \in V^{W}\right)$ or $\left(u \in V^{W}\right.$ and $\left.v \in V^{B}\right)$ : such a graph is often denoted as $G=\left(V^{B} \cup V^{W}, E\right)$. If $G$ is bipartite and we set $V_{B}=V^{B}$ and $V_{W}=V^{W}$ in $k$ ECABP then $G^{\prime}$ is bipartite.

This problem, denoted as B- $k \mathrm{ECABP}$, is a typical subproblem of $k \mathrm{ECABP}$, where "B-" means that $G$ is a bipartite graph. There are several applications for security of statistical data stored in a cross tabulated table [5], and so on.

[Existing Results] Many algorithms for $k \mathrm{ECA}$ have been

Manuscript received April 12, 2011.

Manuscript revised July 16, 2011.

${ }^{\dagger}$ The authors are with the Graduate School of Engineering, Hiroshima University, Higashihiroshima-shi, 739-8527 Japan.

${ }^{\dagger}$ The author is with the Department of Information and Communications Technology, Faculty of Engineering, Hiroshima International University, Kure-shi, 737-0112 Japan.

a)E-mail: oki@infonets.hiroshima-u.ac.jp

DOI: 10.1587/transinf.E95.D.769 proposed. [3] proposed a linear time algorithm for 2ECA, and [4], [9], [15] proposed polynomial time algorithms for $k$ ECA. [5] proposed a linear time algorithm for B-2ECABP, and an $O(\log |V|)$ parallel time algorithm on an EREW PRAM with a linear number of processors. [1] proposed an $O(|V|(|E|+|V| \log |V|) \log |V|)$ time algorithm for $k$ ECAMP that is $k$ ECA with $r$-partition constraints, where $r$-partition $\pi_{M}=\left\{V_{1}, \ldots, V_{r}\right\}(r \geq 2)$ of $V$ is given and $E_{f}$ consists of edges connecting between $V_{i}$ and $V_{j}(i \neq j)$. Note that, in [1], a given multigraph is handled as an edge-weighted simple one such that, for any pair of vertices $u$ and $v$, if there are $x$ multiple edges between $u$ and $v$ then it is represented as a simple edge $(u, v)$ with a weight $w((u, v))=x$. Let M- $k$ ECAMP denote $k$ ECAMP in which $G$ is an $r$-paritite graph, where "M-" means a multipartite graph. Recently, [2] proposed a linear time algorithm for 2ECAMP, and an $O(\log |V|)$ parallel time algorithm on an EREW PRAM with a linear number of processors.

[The Main Results] The main result of the paper is to propose a fast algorithm for obtaining an optimal solution to $(\sigma+1)$ ECABP in linear time when $G$ is $\sigma$-edgeconnected and a structural graph $F(G)$ of $G$ is given, where a structural graph $F(G)$ represents all minimum cuts of $G$. The time complexity of the proposed algorithm is $O\left(|V||E|+|V|^{2} \log |V|\right)$ because $F(G)$ can be constructed in $O\left(|V||E|+|V|^{2} \log |V|\right)$ time [10]. Moreover, it follows from our result that, when $\sigma \in\{1,2\}$, the problem can be solved in linear time because $F(G)$ can be constructed in $O(|V|+|E|)$ time [8], [12]. Note that the proposed algorithm is faster than the algorithm proposed in [1] for $(\sigma+1)$ ECAMP when

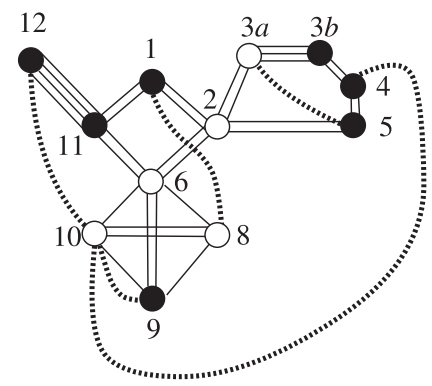

Fig. 1 A multigraph $G$ with $\lambda(G)=4$, where a closed circle (an open circle, respectively) represents a vertex which belongs to $V_{B}\left(V_{W}\right)$, where the corresponding edge-weighted simple graph is used in actual handling. The set of dashed lines represents an optimal solution $E_{f}=\{(1,8),(3 a, 5)$, $(4,10),(9,10),(10,12)\}$ to $5 \mathrm{ECABP}$. 
$r=2$ and $G$ is $\sigma$-edge-connected.

[The Structure of the Paper] The paper is organized as follows. Section 2 provides some definitions and notations. Section 3 shows a lower bound on the cardinality of optimal solutions to this problem. Section 4 presents an algorithm for finding an optimal solution to this problem. We consider its correctness and time complexity in Sects. 4.3 and 4.4, respectively. The concluding remarks are given in Sect. 5 .

\section{Definitions}

[Basic Terminologies of Graphs] An undirected multigraph is denoted as $G=(V(G), E(G))$, where $V(G)$ and $E(G)$ are often denoted as $V$ and $E$, respectively. In this paper, only graphs without loops are considered, and the term "a graph" means an undirected multigraph unless otherwise stated.

Remark 1 . It should be noted that conceptually a multigraph is considered, while the corresponding edge-weighted simple graph is used in actual handling in this paper.

An edge that is incident to two vertices $u, v$ in $G$ is denoted by $(u, v)$. For two disjoint sets $X, X^{\prime} \subset V$, we denote $\left(X, X^{\prime} ; G\right)=\left\{(u, v) \in E \mid u \in X\right.$ and $\left.v \in X^{\prime}\right\}$, where it is often written as $\left(X, X^{\prime}\right)$ if $G$ is clear from the context. We denote $d_{G}(X)=|(X, V-X ; G)|$ which is called the degree of $X$ (in $G)$. For a vertex $v$, the total number of edges incident to $v$ is called the degree of $v$ and is denoted as $d_{G}(v)$ (in $G$ ). The set $(X, V-X ; G)$ is called a $k$-cut when $|(X, V-X)|=k$. For an edge set $E_{f}$, let $G+E_{f}$ denote the graph $\left(V, E \cup E_{f}\right)$. For a given bipartition $\pi=\left(V_{B}, V_{W}\right)$ of $V$ with $V_{B} \cap V_{W}=\emptyset, E_{f}$ is said to be legal (with respect to $\pi$ ) if $E_{f}$ consists of edges connecting between $V_{B}$ and $V_{W}$. If $\pi$ is fixed then "with respect to $\pi$ " is omitted.

A trail is a sequence of distinct edges $\left(v_{0}, v_{1}\right)$, $\left(v_{1}, v_{2}\right), \ldots,\left(v_{r-1}, v_{r}\right)$ in which there may appear the same endvertices, and each of $v_{0}$ and $v_{r}$ is called a terminal vertex of the trail. It is called a closed trail when $v_{0}=v_{r}$. A closed trail is called an Eulerian closed trail of $G$ if all edges of $G$ are included. A path is a trail such that all vertices $v_{0}, v_{1}, \ldots, v_{r}$ are distinct. A cycle consists of a path with $r \geq 1$ and an edge $\left(v_{r}, v_{0}\right)$.

We say that $G$ is connected if there is a path between any pair of vertices. For two vertices $u, v \in V$, let $\lambda(u, v ; G)$ denote the maximum number of edge-disjoint paths between $u$ and $v$ in $G$. The edge-connectivity $\lambda(G)$ of $G$ is defined by $\lambda(G)=\min \{\lambda(u, v ; G) \mid u, v \in V\}$, and we say that $G$ is $k$ edge-connected if $\lambda(G) \geq k$ for a nonnegative integer $k$. If a set $Z \subseteq V$ is a maximal vertex set such that $\lambda(u, v ; G) \geq$ $k$ holds for any pair of vertices $u, v \in Z$ then $Z$ is called a $k$-edge-connected component ( $k$-component, for short) of $G$. For a $k$-component $Z, Z$ is called a leaf $k$-component if $d_{G}(Z)=\lambda(G)$. Note that distinct $k$-components are pairwise disjoint.

[Structural Graphs with Bipartition Constraints] A cactus is an undirected connected graph in which any pair of cycles share at most one vertex. A structural graph $F(G)=$ $(V(F(G)), E(F(G)))[6]$ of $G$ with $\lambda(G)=\sigma$ (see Fig. 2) is a

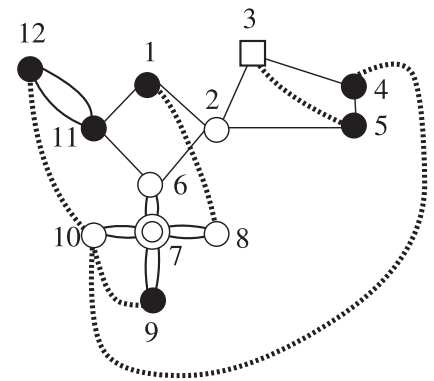

Fig. 2 The set of dashed lines represents an optimal solution $E^{\prime}=\{(1,8)$, $(3,5),(4,10),(9,10),(10,12)\}$ for a structural graph $F(G)$ of $G$ in Fig. 1, giving us $\lambda\left(F(G)+E^{\prime}\right)=\lambda(F(G))+1$.

representation for all minimum cuts of $G$. $F(G)$ is an edgeweighted cactus of $O(|V|)$ vertices and edges such that each tree edge (a bridge in $F(G)$ ) has weight $\lambda(G)$ and each cycle edge (an edge included in a cycle) has weight $\lambda(G) / 2$. Particularly if $\lambda(G)$ is odd then $F(G)$ is an edge-weighted tree. Each vertex in $G$ maps to exactly one vertex in $F(G)$. Note that any minimum cut of $G$ is represented as either a tree edge or a pair of two cycle edges in the same cycle of $F(G)$, and vice versa. Let $\rho: V(G) \rightarrow V(F(G))$ denote this mapping. We use the following notations: $\rho(X)=\{\rho(v) \mid v \in X\}$ for $X \subseteq V$ and $\rho^{-1}(Y)=\{v \in V \mid \rho(v) \in Y\}$ for $Y \subseteq V(F(G)$ ). A vertex $y \in V(F(G))$ with $\rho^{-1}(y)=\emptyset$ is called an empty vertex. Let $\varepsilon(G) \subseteq V(F(G))$ denote the set of all empty vertices of $F(G)$.

For any cut $(X, V(F(G))-X ; F(G))$, if the summation of weights of all edges in the cut is equal to $\sigma$ then $\left(\rho^{-1}(X), V-\rho^{-1}(X) ; G\right)$ is a $\sigma$-cut (a minimum cut) of $G$. Conversely, for any $\sigma$-cut $(X, V-X ; G), F(G)$ has at least one cut $(Y, V(F(G))-Y ; F(G))$ in which the summation of weight of all edges in the cut is equal to $\sigma$, where $Y$ is a vertex set of $G$ such that $\rho(X)=Y-\varepsilon(G)$. Each $(\sigma+1)$-component $S$ of $G$ is represented as a vertex $\rho(S) \in V(F(G))-\varepsilon(G)$ of $F(G)$, and, for any vertex $v \in V(F(G))-\varepsilon(G)$ of $F(G)$, $\rho^{-1}(v)$ is a $(\sigma+1)$-component of $G$. In particular, for $v \in V(F(G))-\varepsilon(G)$ of $F(G)$, we often denote a vertex that is included in a $(\sigma+1)$-component $\rho^{-1}(v)$ as $n_{v}$. For a vertex $v \in V(F(G))-\varepsilon(G)$ of $F(G)$, if the summation of weights of all edges that are incident to $v$ in $F(G)$ equals to $\sigma$, then $v$ is called a leaf of $F(G)$ and $\rho^{-1}(v)$ is a leaf $(\sigma+1)$-component of $G$. Conversely, for any leaf $(\sigma+1)$-component $L$ of $G$, $\rho(L)$ is a leaf of $F(G)$. Note that any leaf of $F(G)$ is not an empty vertex. Let $L F(G)$ denote the set of all leaves of $F(G)$. It is shown in [10] that $F(G)$ can be constructed in $O\left(|V||E|+|V|^{2} \log |V|\right)$ time.

If $F(G)$ has any bridge of weight $\lambda(G)$ then we replace such a bridge by a pair of multiple edges, assigning each edge weight $\lambda(G) / 2$ even if $\lambda(G)$ is odd. We consider such a pair of multiple edges to be a cycle of length two. We call this graph a modified cactus, and we assume $F(G)$ is a modified one in this paper unless otherwise stated. Note that we can handle this modified cactus as a structural graph of $G$ and $\lambda(F(G))=2$.

Even if $\lambda(G)$ is odd, finding an edge set $E_{f}$ such that 
$\lambda\left(G+E_{f}\right)=\lambda(G)+1$ can be solved by fixing an edge set $E^{\prime}$ such that $\lambda\left(F(G)+E^{\prime}\right)=3$ for a modified cactus $F(G)$ of $G$. This is because there is a bijection $\xi: E^{\prime} \rightarrow E_{f}$ such that $\xi((u, v)) \rightarrow\left(n_{u}, n_{v}\right)$, where $\rho\left(n_{u}\right)=u$ and $\rho\left(n_{v}\right)=v$.

Given a structural graph $F(G)$ of a graph $G=(V, E)$ with bipartition constraints $\pi=\left\{V_{B}, V_{W}\right\}$, we classify vertices $v \in V(F(G))-\varepsilon(G)$ into three members as follows:

(i) $\rho^{-1}(v) \subseteq V_{B}(v$ is called a black vertex of $F(G)$ );

(ii) $\rho^{-1}(v) \subseteq V_{W}(v$ is called a white one of $F(G)$ );

(iii) $\rho^{-1}(v) \cap V_{B} \neq \emptyset$ and $\rho^{-1}(v) \cap V_{W} \neq \emptyset(v$ is called a hybrid one of $F(G)$ ).

Each vertex of $V_{B}$ or of $V_{W}$ is called a black vertex or a white one of $G$, respectively. The set of black leaves, white leaves or hybrid leaves of $F(G)$ is denoted by $B F(G), W F(G)$ or $H F(G)$, respectively. In this paper, without loss of generality, we assume that $V_{B} \neq \emptyset, V_{W} \neq \emptyset$ and $|B F(G)| \geq|W F(G)|$. If $|B F(G)|>|W F(G)|+|H F(G)|$ holds then $F(G)$ is said to be $B$-dominant [5].

In figures of this paper, a hybrid vertex is represented by a square, and a black one, a white one and an empty one are represented by a closed circle, an open one and a double one, respectively (see Fig. 2).

\section{A Lower Bound of a Feasible Solution to $(\sigma+$ 1)ECABP}

In the rest of the paper, we set $\sigma=\lambda(G)$.

In this section, a lower bound of a feasible solution to $(\sigma+1)$ ECABP is given. Since $(\sigma+1)$ ECABP is a subproblem of $k$ ECAMP, we obtain the following proposition by setting $k=\sigma+1$ and $r=2$ for a lower bound shown in [1] on $k$ ECAMP.

Suppose $F(G)$ has a sequence of $r$ pairs $(r \geq 1)$ of multiple edges (consisting of a cycle of length 2) from a leaf $u_{0}$ to a vertex $u_{r}$ that is included in a cycle of length at least

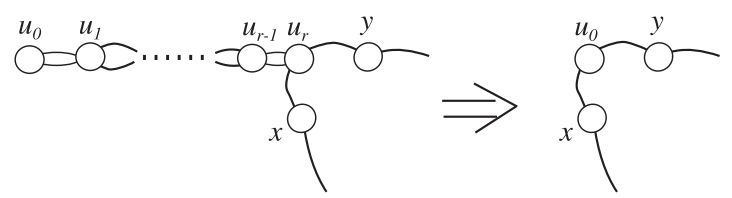

Fig. 3 Schematic explanation of leaf-pruning from a leaf $u_{0}$ to a vertex $u_{r}(r \geq 1)$ of $F(G)$ in a cycle of length at least three of $F(G)$.

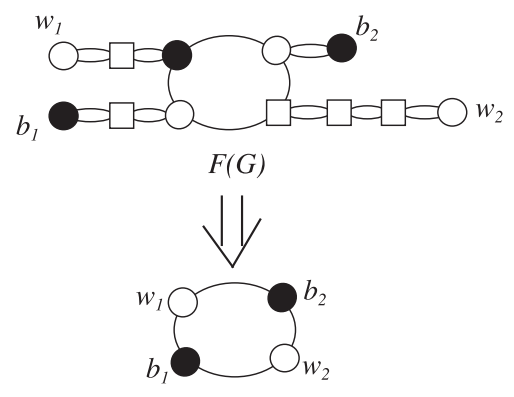

$G_{c}$

Fig. 4 An example of constructing $G_{c}$ from a given $F(G)$ (by repeating leaf-pruning). three. Then let us shrink all vertices $u_{0}, \ldots, u_{r-1}$ to $u_{r}$ and remove any resulting self-loop, and then rename $u_{r}$ as $u_{0}$. If $u_{0}$ is a black or a white or a hybrid vertex then so is the renamed vertex $u_{0}$. We call this operation leaf-pruning (from $u_{0}$ to $u_{r}$ ). Figure 3 shows schematic explanation of leafpruning.

Proposition 3.1 A lower bound $\mathcal{L}$ on the number of edges required to $(\sigma+1)$-edge-connect a given $\sigma$-edge-connected graph $G$ with bipartition constraints is stated in (i) or (ii). (i) Let $G_{c}$ be a graph obtained from $F(G)$ by repeating leaf-pruning (see Fig.4). If this graph $G_{c}$ is a simple cycle of length four such that two black leaves and two other ones that are either white or hybrid appear alternately (see Fig. 4) then $\mathcal{L}=3$. (ii) Otherwise, $\mathcal{L}=$ $\max \{|B F(G)|,|W F(G)|,\lceil|L F(G)| / 2\rceil\}$.

An algorithm to be proposed in the next section finds an edge set whose cardinality is equal to the lower bound of Proposition 3.1, showing that the algorithm finds an optimal solution.

\section{A Proposed Algorithm for $(\sigma+1)$ ECABP}

In this section, we propose a fast algorithm for $(\sigma+$ 1)ECABP.

\subsection{An Outline of the Proposed Algorithm}

From properties of a structural graph, it is enough to consider 3ECABP for $F(G)$ instead of $(\sigma+1)$ ECABP for $G$ with $\lambda(G)=\sigma$ (see [7], [11]). We call an optimal solution to 3ECABP for $F(G)$ an optimal solution to $F(G)$. Without loss of generality, we can focus on the case where $F(G)$ is a modified cactus with $\lambda(F(G))=2$.

In order to efficiently augment the connectivity of $G$ by one, we require that an optimal solution to $F(G)$ should consist of edges $(u, v)$ connecting as many leaves as possible. Furthermore, in order to keep bipartition constraints, the corresponding vertex $n_{u}$ ( $n_{v}$, respectiely) of $G$ should be a black vertex (a white one) in $\rho^{-1}(u)\left(\rho^{-1}(v)\right)$.

We outline how to find an optimal solution to $F(G)$. First, in order to narrow the gap between the number of black leaves and that of white ones, each hybrid leaf is regarded as a black leaf or a white one because any hybrid leaf can be treated as a black or a white one. Next, we repeat an operation to decrease the number of leaves by adding an edge connecting a black leaf and a white one. However, if $F(G)$ is $B$-dominant then, after some iterations, there appears the situation where no white leaves are left. Then we find edges each of which connects a black leaf and either a white vertex or a hybrid one (which is not a leaf). Finally, we obtain an optimal solution to $F(G)$ and then we convert it into an optimal solution to $G$.

Now we explain a special type of preorder of a modified cactus $F(G)$. This is introduced in [11] for finding an optimal solution to $F(G)$ efficiently. First, we assign all 
simple cycles in the cactus distinct colors. Note that this "color" is different from a color "black" or "white" to represent partition constraints. This coloring can be done in $O(|V|+|E|)$ time utilizing a depth-first search. Next, another depth-first-search starts at an arbitrary vertex in the following manner: if any vertex $u$ is visited the first time via an edge in some simple cycle (for example, its color is red) then, before traversing another edge which is in the red cycle and incident to $u$, the other edges incident to $u$ are traversed. (Vertices of $F(G)$ in Fig. 2 are numbered according to this preorder.) This search assigns preorder (denoted as $\beta(v))$ to each vertex $v$ from 1 to $|V(F(G))|$. Note that traversing vertices in the order of $\beta(v)$ from 1 to $|V(F(G))|$ makes an Eulerian closed trail $E T(F(G))$ of $F(G)$.

\subsection{Description of the Algorithm}

The proposed algorithm to find an optimal solution is described as follows.

Algorithm $S_{0} l_{-}(\sigma+1)$ ECABP

Input: A connected graph $G=(V, E)$ with bipartition constraints $\pi=\left\{V_{B}, V_{W}\right\}$.

Output: An edge set $E_{f}$ with minimum cardinality such that $\left(V, E \cup E_{f}\right)$ is $(\sigma+1)$-edge-connected and $E_{f}$ is legal (with respect to $\pi$ ).

1: Construct a structural graph $F(G)=(V(F(G)), E(F(G)))$;

2: Compute a special type of preorder $\beta(v)$ for any vertex $v$ of $F(G)$;

3: $E^{\prime} \leftarrow \emptyset, E_{1}^{\prime} \leftarrow \emptyset, E_{2}^{\prime} \leftarrow \emptyset, E_{3}^{\prime} \leftarrow \emptyset, B \leftarrow B F(G)$, $W \leftarrow W F(G)$, and $H \leftarrow H F(G)$;

4: if $H \neq \emptyset$ then

5: $\quad$ Choose $\min \{\lfloor(|L F(G)|-2|W|) / 2\rfloor,|H|\}$ hybrid leaves arbitrarily, add them to $W$ (regarded as white leaves), and add the other hybrid ones to $B$ (regarded as black leaves) if they exist;

6: $\quad H \leftarrow \emptyset ; / *$ After this step, we have both $|B| \geq|W|$ (because $|W| \leq\lfloor|L F(G)| / 2\rfloor$ ) and $|L F(G)|=|B|+|W|$. */

7: end if

8: while $|B \cup W| \geq 5$ and $W \neq \emptyset$ do

9: $\quad$ Choose a black leaf $b$ and a white leaf $w$ according to Lemma 4.7 if $|B|=|W|=3$, or according to Lemma 4.6 otherwise; $E_{1}^{\prime} \leftarrow E_{1}^{\prime} \cup\{(b, w)\}, B \leftarrow B-\{b\}$ and $W \leftarrow W-\{w\} ;$

10: end while

11: if $|B \cup W| \geq 5$ and $W=\emptyset$ then

12: Find any vertex $w$ which is a white one or a hybrid one of $F(G)$;

13: $\quad E_{2}^{\prime} \leftarrow E_{2}^{\prime} \cup\{(b, w) \mid b \in B\}$ and $B \leftarrow \emptyset$; /* See Lemma 4.2.*/

14: end if

15: if $|B \cup W|=4$ then

16: Find an edge set $E_{3}^{\prime}$ according to Lemma 4.5;

17: else if $|B \cup W|=3$ then

18: $\quad$ Find an edge set $E_{3}^{\prime}$ according to Lemma 4.4;

19: else if $|B \cup W|=2$ then

20: $\quad$ Find an edge set $E_{3}^{\prime}$ according to Lemma 4.3;

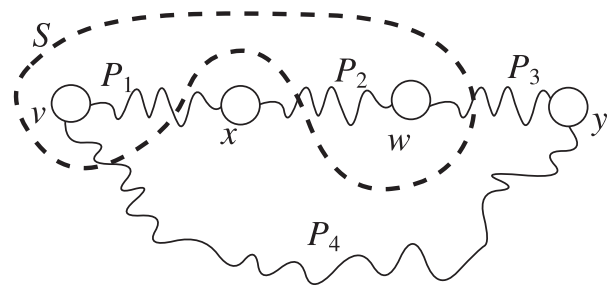

Fig. 5 Schematic explanation of four vertices $v, w, x$ and $y$ included in ET $(F(G))$ of Lemma 4.1.

21: end if

22: $E^{\prime} \leftarrow E_{1}^{\prime} \cup E_{2}^{\prime} \cup E_{3}^{\prime}$;

23: Output $E_{f}=\left\{\left(n_{b}, n_{w}\right) \mid(b, w) \in E^{\prime}\right\}$;

\subsection{Correctness of the Algorithm}

We prove correctness of the algorithm by using several lemmas and a theorem. $F(G)$.

First, we show the next lemma for a structural graph

Lemma 4.1 Suppose $|L F(G)| \geq 4$ for a structural graph $F(G)$. If there are distinct four leaves $v, w, x, y$ of $F(G)$ with $\beta(v)<\beta(x)<\beta(w)<\beta(y)$ then we can choose four vertices $n_{v}, n_{w}, n_{x}, n_{y} \in V(G)$ such that $\left|L F\left(G+\left\{\left(n_{v}, n_{w}\right)\right\}\right)\right|=$ $|L F(G)|-2$ and $\left|L F\left(G+\left\{\left(n_{x}, n_{y}\right)\right\}\right)\right|=|L F(G)|-2$.

Proof. An Eulerian closed trail $E T(F(G))$ can be determined by traversing vertices of $F(G)$ in the order of $\beta(v)$ assigned to each vertex $v \in V(F(G))$. Let us separate $\operatorname{ET}(F(G))$ into trails $P_{1}, P_{2}, P_{3}$ and $P_{4}$ such that the pairs of terminal vertices are $\{v, x\},\{x, w\},\{w, y\}$ and $\{y, v\}$, respectively (see Fig. 5). Clearly they are pairwise edge-disjoint.

Now we count the number of leaves of $F\left(G+\left\{\left(n_{v}, n_{w}\right)\right\}\right)$. Let $S \subset V(F(G))$ be any set such that $\{v, w\} \subseteq S$ and $\{x, y\} \subseteq V(F(G))-S$. Since $F(G)$ has four trails $P_{i}(1 \leq$ $i \leq 4)$ and $d_{F(G)}(S) \geq 4,(S, V(F(G))-S)$ is not a 2-cut of $F(G)$. It follows from properties of a structural graph that $\left(\rho^{-1}(S), V-\rho^{-1}(S)\right)$ is not a $\sigma$-cut of $G$. Hence $\rho^{-1}(S)$ in $G+\left\{\left(n_{v}, n_{w}\right)\right\}$ cannot be a leaf $(\sigma+1)$-component, showing that $\left|L F\left(G+\left\{\left(n_{v}, n_{w}\right)\right\}\right)\right|=|L F(G)|-2$. Similarly, we can prove that $\left|L F\left(G+\left\{\left(n_{x}, n_{y}\right)\right\}\right)\right|=|L F(G)|-2$.

A pair $\{v, w\}$ (or a pair $\{x, y\}$ ) appeared in Lemma 4.1 is called an augmenting pair with respect to $v, w, x$ and $y$.

In the rest of this section, we discuss properties for bipartition constraints. We show the next lemma for a black leaf $b$ and either a white one or a hybrid one $w$ used in Lemmas $4.3-4.5$ and Step 11 of Sol_$(\sigma+1)$ ECABP.

Lemma 4.2 Suppose $W F(G) \cup H F(G)=\emptyset$ and $|B F(G)| \geq$ 2. Then there exists an optimal solution $E_{f}=\left\{\left(n_{b}, n_{w}\right) \mid b \in\right.$ $B F(G)\}$ with $\left|E_{f}\right|=|B F(G)|$ and $\lambda\left(G+E_{f}\right)=\sigma+1$, where $w$ is any white vertex or hybrid one (that is not a leaf) of $F(G)$.

Proof. Let us consider an edge set $E^{\prime}=\{(b, w) \mid b \in B F(G)\}$. Then $\lambda\left(b_{1}, b_{2} ; F(G)+E^{\prime}\right)=3$ for any distinct two black leaves $b_{1}$ and $b_{2}$, since $\left\{\left(b_{1}, w\right),\left(b_{2}, w\right)\right\} \subseteq E^{\prime}$ and $F(G)+E^{\prime}$ 
has a path connecting $b_{1}$ and $b_{2}$ and consisting of two edges $\left(b_{1}, w\right)$ and $\left(b_{2}, w\right)$. Thus, by Proposition 3.1, $E_{f}=\left\{\left(n_{b}, n_{w}\right) \mid\right.$ $b \in B F(G)\}$ is an optimal solution to $G$ and $\left|E_{f}\right|=|B F(G)|$.

If $|L F(G)| \leq 4$ then we can show the next Lemmas 4.34.5 , where they are used in Steps 15-19 of the proposed algorithm.

Here we summarize cardinalities of $L F(G), B F(G)$, $W F(G)$ and $H F(G)$, as well as $\Delta=\min \{[|L F(G)|-$ $2|W F(G)|) / 2\rceil,|H F(G)|\}$ and $\mathcal{L}=\max \{|B F(G)|,|W F(G)|$, $\lceil|L F(G)| / 2\rceil\}$ in Table 1. They are used in proofs of Lemmas 4.3, 4.4 and 4.5 .

In the rest of this section let us consider situations after Step 6 of Sol $_{-}(\sigma+1)$ ECABP.

Lemma 4.3 Suppose $|L F(G)|=2$ for $F(G)$. Then there exists an optimal solution $E_{f}$ with $\left|E_{f}\right|=2$ if $|B F(G)|=2$, or $\left|E_{f}\right|=1$ if $|B F(G)| \leq 1$.

Proof. If $|B F(G)|=2$ then the lemma follows from Lemma 4.2. If $|B F(G)| \leq 1$ then $\mathcal{L}=1$, and Table 1 shows that we can choose a black vertex $n_{b}$ and a white one $n_{w}$, where $n_{b}$ and $n_{w}$ are included in $\rho^{-1}(b)$ and $\rho^{-1}(w)$ for the two leaves $b \in B \subseteq B F(G) \cup H F(G)$ and $w \in W \subseteq W F(G) \cup$ $H F(G)$, respectively. Clearly $\lambda\left(G+\left\{\left(n_{b}, n_{w}\right)\right\}\right)=\sigma+1$. The

Table 1 Summary of cardinalities of sets and several values appearing in Lemmas 4.3, 4.4 and 4.5 .

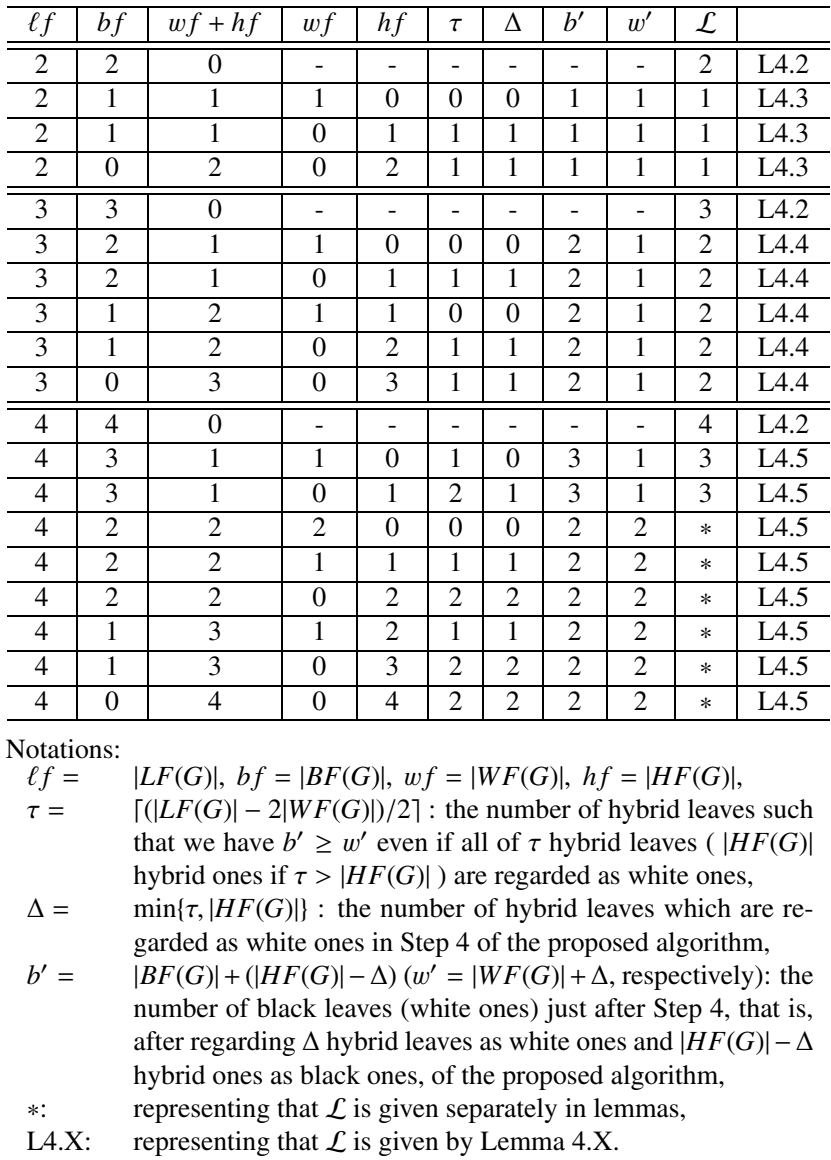

lemma follows from Proposition 3.1.

Lemma 4.4 Suppose $|L F(G)|=3$ for $F(G)$. Then there exists an optimal solution $E_{f}$ with $\left|E_{f}\right|=3$ if $|B F(G)|=3$, or $\left|E_{f}\right|=2$ if $|B F(G)| \leq 2$.

Proof. If $|B F(G)|=3$ then the lemma follows from Lemma 4.2. Next, we consider the case with $|B F(G)| \leq 2$. Then $\mathcal{L}=2$ and Table 1 shows that we can choose either (i) two black vertices $n_{b_{1}}, n_{b_{2}}$ and one white one $n_{w}$ that are included in $\rho^{-1}\left(b_{1}\right), \rho^{-1}\left(b_{2}\right)$ and $\rho^{-1}(w)$ for the three leaves $b_{1}, b_{2} \in B \subseteq B F(G) \cup H F(G)$ and $w \in W \subseteq W F(G) \cup$ $H F(G)$, respectively; or (ii) one black vertex $n_{b}$ and two white ones $n_{w_{1}}, n_{w_{2}}$ that are included in $\rho^{-1}(b), \rho^{-1}\left(w_{1}\right)$ and $\rho^{-1}\left(w_{2}\right)$ for the three leaves $b \in B \subseteq B F(G) \cup H F(G)$ and $w_{1}, w_{2} \in W \subseteq W F(G) \cup H F(G)$, respectively. Clearly $\lambda\left(G+\left\{\left(n_{b_{1}}, n_{w}\right),\left(n_{b_{2}}, n_{w}\right)\right\}\right)=\sigma+1$ in the case (i), or $\lambda\left(G+\left\{\left(n_{w_{1}}, n_{b}\right),\left(n_{w_{2}}, n_{b}\right)\right\}\right)=\sigma+1$ in the case (ii). The lemma follows from Proposition 3.1.

Lemma 4.5 Suppose $|L F(G)|=4$ for $F(G)$.

(i) If $|B F(G)| \geq 3$ then there exists an optimal solution $E_{f}$ with $\left|E_{f}\right|=|B F(G)|$.

(ii) If $|B F(G)| \leq 2$ then let us consider a graph $G_{c}$ defined in Proposition 3.1.

(ii-1) If $G_{c}$ is a simple cycle of length four such that two black leaves and two other ones that are either white or hybrid ones appear alternately (see Fig. 6) then there exists an optimal solution $E_{f}$ with $\left|E_{f}\right|=3$;

(ii-2) otherwise, there exists an optimal solution $E_{f}$ with $\left|E_{f}\right|=2$.

Proof. We consider the two cases (i) and (ii) separately.

[The case (i)] If $|B F(G)|=4$ then the lemma follows from Lemma 4.2. If $|B F(G)|=3$ then, by applying Lemma 4.1 to the four leaves, we can obtain an augmenting pair of $\{b, w\}$, where $b$ is a black leaf and $w$ is either a white one or a hybrid one with respect to the four ones. $F\left(G+\left\{\left(n_{b}, n_{w}\right)\right\}\right)$ has two black leaves. The lemma follows from Lemma 4.2 and Proposition 3.1.

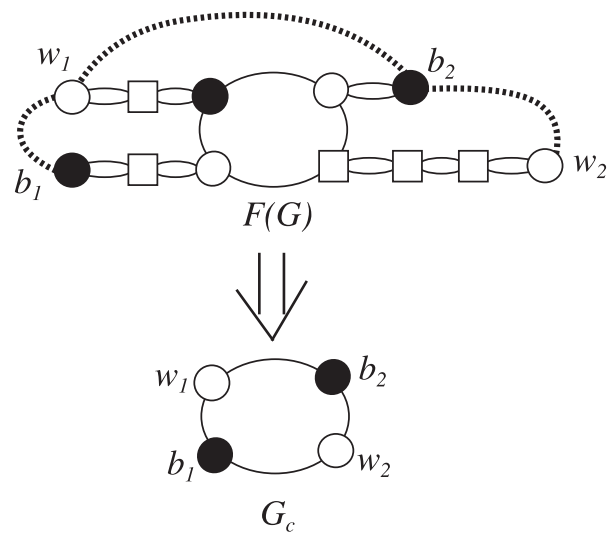

Fig. 6 Schematic explanation of Lemma 4.5, where dash lines represent an optimal solution 
[The case (ii)] There are the two subcases (ii-1) and (ii-2).

(The case (ii-1)) Clearly the lemma follows from Proposition 3.1.

(The case (ii-2)) Table 1 shows that we can choose an augmenting pair $\{b, w\}$ with respect to the four leaves such that there are a black vertex $n_{b} \in \rho^{-1}(b)$ and a white one $n_{w} \in \rho^{-1}(w)$ so that $F\left(G+\left\{\left(n_{b}, n_{w}\right)\right\}\right)$ may have two leaves including at most one black one. The lemma follows from Proposition 3.1 and Lemma 4.3.

Next, we consider the case with $|L F(G)| \geq 5$. We show the following two lemmas for a black leaf $b$ and a white one $w$ in Step 8 of Sol_$(\sigma+1)$ ECABP. For simplicity of description, let us call a hybrid leaf $v \in B$ ( $v \in W$, respectively) at Step 8 a black leaf (a white one) in the rest of this section, and without loss of generality we denote $B=\left\{b_{1}, \ldots, b_{|B|}\right\}$ and $W=\left\{w_{1}, \ldots, w_{|W|}\right\}$, where $\beta\left(b_{1}\right)<\cdots<\beta\left(b_{|B|}\right)$ and $\beta\left(w_{1}\right)<\cdots<\beta\left(b_{|W|}\right)$.

Lemma 4.6 Suppose $|B \cup W| \geq 5$ and $W \neq \emptyset$ in Step 8 . Then there is a set of four leaves from which we can choose a black leaf $b$ and a white one $w$ of $F(G)$ such that $\{b, w\}$ is an augmenting pair with respect to the four ones.

Proof. We prove the lemma by showing an augmenting pair of a black leaf and a white one with respect to some four ones in $B \cup W$. Let us consider the two cases (i) $|W|=1$ and (ii) $|W|>1$, separately.

[The case (i)] We consider the four subcases (i-1)-(i4 ) according to the sequences of four leaves $w_{1}, b_{1}, b_{2}$ and $b_{3}$ sorted in increasing order of $\beta$. Then we can select an augmenting pair with respect to the four leaves as follows: (i-1) if $\beta\left(w_{1}\right)<\beta\left(b_{1}\right)<\beta\left(b_{2}\right)<\beta\left(b_{3}\right)$ then select $w_{1}$ and $b_{2}$; (i-2) if $\beta\left(b_{1}\right)<\beta\left(w_{1}\right)<\beta\left(b_{2}\right)<\beta\left(b_{3}\right)$ then select $w_{1}$ and $b_{3}$; (i-3) if $\beta\left(b_{1}\right)<\beta\left(b_{2}\right)<\beta\left(w_{1}\right)<\beta\left(b_{3}\right)$ then select $b_{1}$ and $w_{1}$; (i-4) if $\beta\left(b_{1}\right)<\beta\left(b_{2}\right)<\beta\left(b_{3}\right)<\beta\left(w_{1}\right)$ then select $b_{2}$ and $w_{1}$.

[The case (ii)] We consider the six subcases (ii-1)-(ii6 ) according to the sequence of the four leaves $w_{1}, w_{2}, b_{1}$ and $b_{2}$ sorted in increasing order of $\beta$. Then we can select an augmenting pair with respect to the four leaves for the first four cases as follows:

(ii-1) if $\beta\left(w_{1}\right)<\beta\left(w_{2}\right)<\beta\left(b_{1}\right)<\beta\left(b_{2}\right)$ then select $w_{1}$ and $b_{1}$;

(ii-2) if $\beta\left(b_{1}\right)<\beta\left(b_{2}\right)<\beta\left(w_{1}\right)<\beta\left(w_{2}\right)$ then select $b_{1}$ and $w_{1}$;

(ii-3) if $\beta\left(w_{1}\right)<\beta\left(b_{1}\right)<\beta\left(b_{2}\right)<\beta\left(w_{2}\right)$ then select $w_{1}$ and $b_{2}$;

(ii-4) if $\beta\left(b_{1}\right)<\beta\left(w_{1}\right)<\beta\left(w_{2}\right)<\beta\left(b_{2}\right)$ then select $b_{1}$ and $w_{2}$.

(ii-5) Now consider the sequence with $\beta\left(w_{1}\right)<\beta\left(b_{1}\right)<$ $\beta\left(w_{2}\right)<\beta\left(b_{2}\right)$. Since $|B \cup W| \geq 5$ and $|B| \geq|W|$, there is $b_{|B|}$ such that $\beta\left(b_{2}\right)<\beta\left(b_{|B|}\right)$. Hence the pair $\left\{w_{1}, b_{2}\right\}$ is an augmenting pair with respect to $w_{1}, w_{2}, b_{2}$ and $b_{|B|}$.

(ii-6) Finally consider the sequence with $\beta\left(b_{1}\right)<\beta\left(w_{1}\right)<$ $\beta\left(b_{2}\right)<\beta\left(w_{2}\right)$. Note that since $|B \cup W| \geq 5$ and $|B| \geq|W|$, $b_{3}$ and $b_{|B|}\left(b_{3}=b_{|B|}\right.$ may hold $)$ are different from $b_{1}, w_{1}, b_{2}$ and $w_{2}$. There are two subcases. (ii-6-a) $W=\left\{w_{1}, w_{2}\right\}$ (that is, $\left.w_{2}=w_{|W|}\right)$ and $\beta\left(b_{|B|}\right)<$ $\beta\left(w_{2}\right)$. The pair $\left\{w_{1}, b_{3}\right\}$ is an augmenting pair with respect to $w_{1}, b_{2}, b_{3}$ and $w_{2}$.

(ii-6-b) Any other case except (ii-6-a). There is a leaf $v$ such that $v$ is different from $b_{1}, w_{1}, b_{2}$ and $w_{2}$, and $\beta(v)=\max \left\{\beta\left(b_{|B|}\right), \beta\left(w_{|W|}\right)\right\}$ holds. Then the pair $\left\{b_{1}, w_{2}\right\}$ is an augmenting pair with respect to $b_{1}, b_{2}, w_{2}$ and $v$.

Even if we select two vertices $n_{b} \in \rho^{-1}(b), n_{w} \in \rho^{-1}(w)$ for the pair $b, w$ of Lemma 4.6, we have to avoid the case where $G_{c}$ constructed from $F\left(G+\left\{\left(n_{b}, n_{w}\right)\right\}\right)$ by repeating leaf-pruning satisfies Proposition 3.1 (i). This can be done as stated in Lemma 4.7.

Lemma 4.7 Suppose $|B|=|W|=3$ in Step 8. There are a black leaf $b$ and a white one $w$ satisfying the following two conditions (i) and (ii): (i) the pair $\{b, w\}$ is an augmenting pair; (ii) any $G_{c}$ constructed from $F\left(G+\left\{\left(n_{b}, n_{w}\right)\right\}\right)$ by repeating leaf-pruning does not satisfy Proposition 3.1 (i), that is, even if $G_{c}$ is a cycle of length four, remaining four leaves (two black ones and two white ones) never appear alternately in increasing order of $\beta$.

Proof. We can assume that $\beta\left(b_{1}\right)<\beta\left(w_{1}\right)$ without loss of generality. Then there are ten kinds of sequences consisting of the six leaves sorted in increasing order of $\beta$. Let us focus on an augmenting pair $\left\{b_{1}, w\right\}$ for some white leaf $w$ with respect to some four leaves including $b_{1}$. In this setting, any choice of $\left\{b_{1}, w\right\}$ in the following two sequences (ii-1) and (ii-2) violates the condition (ii) of the lemma, while we can select $\left\{b_{1}, w\right\}$ satisfying the lemma in the remaining eight sequences.

(ii-1) $\beta\left(b_{1}\right)<\beta\left(b_{2}\right)<\beta\left(w_{1}\right)<\beta\left(w_{2}\right)<\beta\left(b_{3}\right)<\beta\left(w_{3}\right)$;

(ii-2) $\beta\left(b_{1}\right)<\beta\left(w_{1}\right)<\beta\left(b_{2}\right)<\beta\left(w_{2}\right)<\beta\left(w_{3}\right)<\beta\left(b_{3}\right)$.

Choosing another pair, however, gives us a desired one. In the case (ii-1), a pair $\left\{b_{2}, w_{3}\right\}$ is an augmenting pair with respect to $b_{1}, b_{2}, w_{1}$ and $w_{3}$, and in the case (ii-2) $\left\{w_{1}, b_{3}\right\}$ is an augmenting pair with respect to $b_{1}, w_{1}, w_{2}$ and $b_{3}$. Moreover, for $F\left(G+\left\{\left(n_{b_{2}}, n_{w_{3}}\right)\right\}\right)$ in (ii-1) and $F\left(G+\left\{\left(n_{b_{3}}, n_{w_{1}}\right)\right\}\right)$ in (ii-2), the remaining four leaves satisfy the condition (ii) of the lemma.

Note that it is not necessary to actually construct a graph $G_{c}$ even if $|B|=|W|=3$ at Step 9.

From Lemmas 4.1-4.7, we obtain the next theorem.

Theorem 4.8 For a given graph $G$ with $\lambda(G)=\sigma$ and a bipartition $\left\{V_{B}, V_{W}\right\}$ of $V$ with $V_{B} \cap V_{W}=\emptyset$, Sol $(\sigma+$ 1)ECABP finds a legal edge set $E_{f}$, with $\left|E_{f}\right|=\mathcal{L}=$ $\max \{|B F(G)|,|W F(G)|,\lceil|L F(G)| / 2\rceil\}$, such that $\lambda\left(G+E_{f}\right)=$ $\sigma+1$.

Proof. Let $b$ ( $w$, respectively) be a black leaf (a white one) which is chosen in Step 8 or 11 . Let $B_{c}$ ( $W_{c}$, respectively) denote the current $B(W)$. For the edge set $E_{f}$ to appear in the proof, its construction shows that it is legal.

For an edge or an edge set found in Steps 8, 11 and 15, we consider the following three cases:

(i) $|L F(G)| \geq 5$ and $|B|=|W|$ just before Step 8; 
(ii) $|L F(G)| \geq 5$ and $|B|>|W|$ just before Step 8 (then $F(G)$ is $B$-dominant);

(iii) $|L F(G)| \leq 4$.

[The case (i)] Note that Step 11 is never executed.

While $|B|=|W|>3$, we can repeat choosing an augmenting pair $\{b, w\}$ by Lemma 4.6. If the case with $|B|=|W|=3$ appears then we can select an augmenting pair $\{b, w\}$ by Lemma 4.7 .

Now, we consider $E_{1}^{\prime}, B_{c}$ and $W_{c}$ just before Step 15. Since Steps 8 and 9 are executed, $\left|E_{1}^{\prime}\right|=|B|-\left|B_{c}\right|=|W|-$ $\left|W_{c}\right|,\left|B_{c}\right|=\left|W_{c}\right|=2$, and $F\left(G+E_{1}\right)$ has four leaves $b_{1}$, $b_{2}, w_{1}$ and $w_{2}$ with $B_{c}=\left\{b_{1}, b_{2}\right\}$ and $W_{c}=\left\{w_{1}, w_{2}\right\}$. Let $E_{1}=\left\{\left(n_{b}, n_{w}\right) \mid(b, w) \in E_{1}^{\prime}\right\}$.

It follows from Lemma 4.7 that two black vertices and two white ones do not appear alternately in any $G_{c}$ constructed from $F\left(G+E_{1}\right)$ by repeating leaf-pruning even if $G_{c}$ is a cycle of length four (see Fig. 6).

Thus there exists an optimal solution $E_{f}$ to $G+E_{1}$ with $\left|E_{f}\right|=2$ by Lemma 4.5 (ii-2), and an edge set $E_{3}^{\prime}$ with $\left|E_{3}^{\prime}\right|=$ 2 is found in Step 15. Hence $\left|E_{f}\right|=|L F(G)| / 2$ and $E_{f}$ is a solution to $G$ by Proposition 3.1.

[The case (ii)] Just after Step 8, one of the following subcases (ii-1)-(ii-4) holds:

(ii-1) $|B|>4$ and $|W|=0$;

(ii-2) $|B|=4$ and $|W|=0$;

(ii-3) $|B|=3$ and $0 \leq|W| \leq 1$;

(ii-4) $|B|=2$ and $|W|=1$.

(The case with $|B|=2$ and $|W|=0$ follows from (ii-3) with $|W|=1$.)

For the case (ii-1), Step 11 is executed and a set $E_{2}^{\prime}$ with $\left|E_{2}^{\prime}\right|=\left|B_{c}\right|$ is obtained. On the other hand, for each of the cases (ii-2)-(ii-4), Step 15 or 17 is executed and a set $E_{3}^{\prime}$ with $\left|E_{3}^{\prime}\right|=\left|B_{c}\right|$ is obtained. Thus $\left|E_{f}\right|=|B F(G)|$.

Concerning the optimal solution $E^{\prime}=E_{1}^{\prime} \cup E_{2}^{\prime} \cup E_{3}^{\prime}$ to $F(G)$ shown in Fig. $2, E_{1}^{\prime}=\{(1,8),(3,5)\}$ is obtained in Step 8 , and $E_{3}^{\prime}=\{(4,10),(9,10),(10,12)\}$ is obtained in Step 15.

[The case (iii)] In Step 15, we can find an edge set $E_{f}$, with $\left.\left|E_{f}\right|=\max \{|B F(G)|,|W F(G)|, \Gamma|L F(G)| / 2\rceil\right\}$, such that $\lambda\left(G+E_{f}\right)=\sigma+1$ by Lemmas 4.3-4.5.

\subsection{Time Complexity}

Let us focus on how to find all edges $(b, w)$ in Step 8 of Sol_ $(\sigma+1)$ ECABP in linear time.

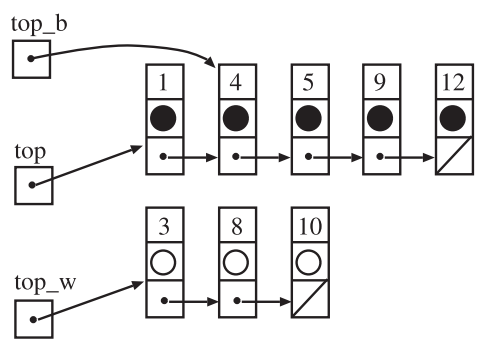

Fig. 7 The data structure used in Step 8. Each element with a black circle (a white one, respectively) represents a black leaf (a white one) of $F(G)$.

[Data Structure] Just before Step 8, we make following preparations (see Fig. 7). We provide two linear lists such that each vertex of $B$ or $W$ is kept in one of the lists in increasing order of $\beta$ (after Step 4, each leaf of $H F(G)$ is included in either $B$ or $W$ ). Each element in the lists has three members, and these three members in the element are denoted as $p \rightarrow \beta, p \rightarrow$ color and $p \rightarrow$ next by using a pointer $p$ to the element of the list. In the element indicated by $p$ and containing a leaf $v$, we store $\beta(v)$ in $p \rightarrow \beta$, its color (black or white) in $p \rightarrow$ color and the pointer to the next element in $p \rightarrow$ next. Note that nil is stored in $p \rightarrow$ next if the element is last one of each list, where it is denoted by a slash in Fig. 7. We also prepare three pointers top, top_b and top_w. For simplicity of description, we often denote the element indicated by a pointer $p$ as a leaf $v$ if $v$ is stored in the element.

The pointer top points to the element containing a leaf with the minimum value of $\beta$ among $B \cup W$. The pointer top_b (top_w, respectively) points to the element containing a leaf with a minimum value of $\beta$ among the set $B(W)$ from which top is removed, where if $W=\emptyset$ then top_w $\leftarrow$ nil.

[The Details of Step 8] We show the detailed description of Step 8 as follows.

$$
\begin{aligned}
& \text { while }|B \cup W| \geq 5 \text { and } W \neq \emptyset \text { do } \\
& \text { if }|B|=|W|=3 \text { then }
\end{aligned}
$$

Let $b$ and $w$ be leaves specified in Lemma 4.7;

else if $|W|=1$ then

Find a black leaf $b$ and a white leaf $w$ by (i-1)-(i-4)

in the proof of Lemma 4.6;

else if top $\rightarrow$ color is black $/ *|W|>1 * /$ then

if $\left\{W=\left\{w_{1}, w_{2}\right\}\right.$ and $\beta\left(b_{|B|}\right)<\beta\left(w_{2}\right)$ and $\beta\left(b_{1}\right)<$ $\left.\beta\left(w_{1}\right)<\beta\left(b_{2}\right)<\beta\left(w_{2}\right)\right\}$ then

Find a black leaf $b$ and a white leaf $w$ by (ii-6-a) in the proof of Lemma 4.6;

Delete the element corresponding to $b_{3}$ (in top $\rightarrow$ next) from the list;

else if top_b $\rightarrow \beta>$ top $\_w \rightarrow \beta$ then

$/ *$ (ii-4) or (ii-6-b) in the proof of Lemma $4.6 * /$

$b \leftarrow$ top; $w \leftarrow$ top_w $\rightarrow$ next;

top $\leftarrow$ top_w; top_w $\leftarrow$ (top_w $\rightarrow$ next $) \rightarrow$ next;

/* top_w may be nil */

else if top_b $\rightarrow \beta<$ top_w $\rightarrow \beta$ then $/ *$ (ii-2) in the proof of Lemma $4.6 * /$ $b \leftarrow$ top; $w \leftarrow$ top_w; top $\leftarrow$ top_b; top_b $\leftarrow$ top_b $\rightarrow$ next; top_w $\leftarrow$ top_w $\rightarrow$ next;

end if

else $/ *$ top $\rightarrow$ color is white and $|W|>1 * /$

if top_b $\rightarrow \beta>$ top_w $\rightarrow \beta$ then $/ *$ (ii-1) in the proof of Lemma $4.6 * /$ $b \leftarrow$ top_b; $w \leftarrow$ top; top $\leftarrow$ top_w; top_w $\leftarrow$ top_w $\rightarrow$ next; top_b $\leftarrow$ top_b $\rightarrow$ next; /* top_w may be nil */

else if top_b $\rightarrow \beta<$ top_w $\rightarrow \beta$ then $/^{*}$ (ii-3) or (ii-5) in the proof of Lemma $4.6 * /$ $b \leftarrow$ top_b $\rightarrow$ next; $w \leftarrow$ top; 


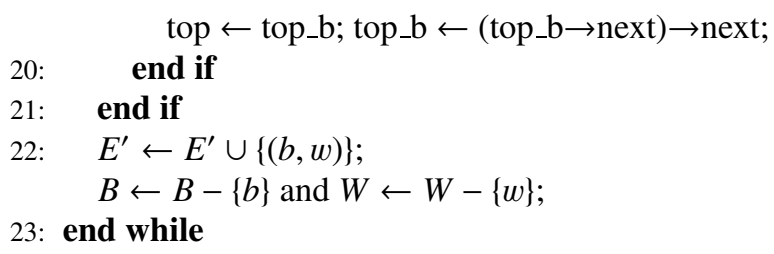

It is straightforward to see that this procedure works correctly.

[Total Time Complexity] Clearly the above procedure can find augmenting pairs shown in Lemma 4.6 or Lemma 4.7 in $O(|V|)$ time. A structural graph can be constructed in $O\left(|V||E|+|V|^{2} \log |V|\right)$ time [10]. Moreover, since all $(\sigma+1)$ components are computed in linear time [8], [12]-[14] when $\lambda(G) \in\{1,2\}$, a structural graph can be constructed in linear time. From above discussion, Proposition 3.1 and Theorem 4.8 , we obtain the next theorem.

Theorem 4.9 Algorithm Sol_ $(\sigma+1)$ ECABP computes an optimal solution to $(\sigma+1)$ ECABP for $G$ with $\sigma=\lambda(G)$ in $O\left(|V||E|+|V|^{2} \log |V|\right)$ time. Moreover, this is reduced to $O(|V|+|E|)$ time when $\sigma \in\{1,2\}$.

\section{Concluding Remarks}

In this paper, we have proposed an $O\left(|V||E|+|V|^{2} \log |V|\right)$ time algorithm to find an optmal solution to $(\sigma+1)$ ECABP when $\sigma=\lambda(G)$. Moreover, we have shown that the problem can be solved in linear time when $\sigma \in\{1,2\}$.

As future research, proposing an efficient algorithm for $(\sigma+1)$ ECAMP with $\sigma=\lambda(G)$ is left.

\section{Acknowledgements}

The research is partly supported by the Grant-in-Aid for Scientific Research (C) (No. 20500015 and 22500029) of the Ministry of Education, Culture, Sports, Science and Technology of Japan.

\section{References}

[1] J. Bang-Jensen, H.N. Gabow, T. Jordán, and Z. Szigeti, "Edgeconnectivity augmentation with partition constraints," SIAM J. Discrete Math., vol.12, no.2, pp.160-207, 1999.

[2] Y. Chen, H. Wei, P. Huang, W. Shih, and T. Hsu, "The bridgeconnectivity augmentation problem with a partition constraint," Theor. Comput. Sci., vol.411, no.31-33, pp.2878-2889, 2010.

[3] K.P. Eswaran and R.E. Tarjan, "Augmentation problems," SIAM J. Comput., vol.5, pp.653-655, 1976.

[4] A. Frank, "Augmenting graphs to meet edge connectivity requirements," SIAM J. Discrete Math., vol.5, no.1, pp.25-53, 1992.

[5] P. Huang, H. Wei, W. Lu, W. Shih, and T. Hsu, "Smallest bipartite bridge-connectivity augmentation," Algorithmica, vol.54, no.3, pp.353-378, 2009.

[6] A.V. Karzanov and E.A. Timofeev, "Efficient algorithm for finding all minimal edge cuts of a nonoriented graph," Cybernetics, pp.156162, March-April 1986, Translated from Kibernetika, 2 (1986).

[7] T. Mashima, S. Taoka, and T. Watanabe, "A 2-approximation algorithm to $(k+1)$-edge-connect a specified set of vertices in a $k$-edgeconnected graph,” IEICE Trans. Fundamentals, vol.E88-A, no.5, pp.1290-1300, May 2005.
[8] H. Nagamochi and T. Ibaraki, "A linear time algorithm for computing 3-edge-connected components in a multigraph," Japan J. Industrial and Applied Math., vol.9, no.7, pp.163-180, 1992.

[9] H. Nagamochi, S. Nakamura, and T. Ibaraki, "A simplified $\tilde{O}(n m)$ time edge-splitting algorithm in undirected graphs," Algorithmica, vol.26, pp.50-57, 2000.

[10] H. Nagamochi, S. Nakamura, and T. Ishii, "Constructing a cactus for minimum cuts of a graph in $O\left(m n+n^{2} \log n\right)$ time and $O(m)$ space," IEICE Trans. Inf. \& Syst., vol.E86-D, no.2, pp.179-185, Feb. 2003.

[11] D. Naor, D. Gusfield, and C. Martel, "A fast algorithm for optimally increasing the edge connectivity," SIAM J. Comput., vol.26, no.4, pp.1139-1165, Aug. 1997.

[12] S. Taoka, T. Watanabe, and K. Onaga, "A linear-time algorithm for computing all 3-edge-connected components of an multigraph," IEICE Trans. Fundamentals, vol.E75-A, no.3, pp.410-424, March 1992.

[13] R.E. Tarjan, "A note on finding the bridges of a graph," Inf. Process. Lett., vol.2, pp.160-161, 1974.

[14] Y.H. Tsin, "Yet another optimal algorithm for 3-edge-connectivity," J. Discrete Algorithms, vol.7, no.1, pp.130-146, 2009.

[15] T. Watanabe and A. Nakamura, "Edge-connectivity augmentation problems," J. Comput. Syst. Sci., vol.35, no.1, pp.96-144, 1987.

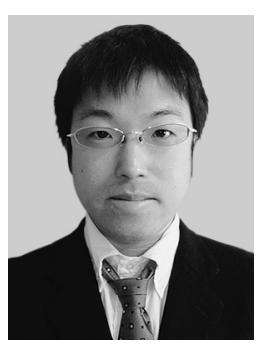

Tadachika Oki was born in Okayama Prefecture on September 8, 1982. He took B.E. from Yamaguchi University in 2005, M.E form Hiroshima University in 2007 and has been a doctor student, working with Prof. T. Watanabe, since April, 2005. His research interest includes graph theories.

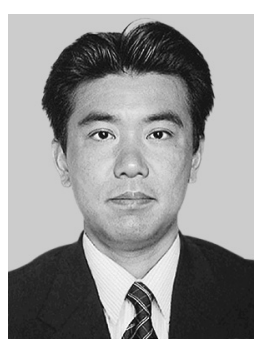

Satoshi Taoka was born in Hiroshima Prefecture on November 28, 1968. He took B.E., M.E. and D.E. degrees from Hiroshima University in 1991, 1994 and 2003 respectively. He is currently a research associate of the Graduate School of Engineering, Hiroshima University. His research interest includes design and analysis of sequential and/or distributed algorithms on graphs and Petri nets. He is a member of the Institute of Electrical and Electronics Engineers (IEEE) and the Institute of Information Processing Society of Japan (IPSJ). 


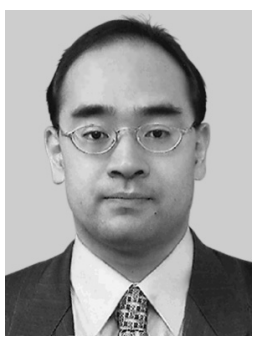

Toshiya Mashima was born in Tokushima Prefecture on November 14, 1969. He took B.E. and M.E. degrees from Hiroshima University in 1992 and 1994, respectively. He is currently a lecturer at the Department of Information and Communications Technology, Faculty of Engineering, Hiroshima International University. His research interest includes design and analysis of graph algorithms. He is a member of the Information Processing Society of Japan and the Operations Research Society of Japan.

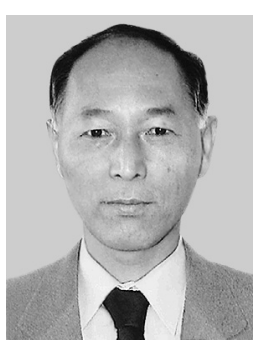

Toshimasa Watanabe was born in Hiroshima Prefecture on January 18, 1949. He took B.E., M.E. and D.E. degrees from Hiroshima University in 1972 and 1974, and from Tohoku University in 1977, respectively. From 1977 to 1981 , he was a research associate at Tohoku University and then at Hiroshimaxq University. From 1981 to 1990 , he was an associate professor at the Department of Applied Mathematics, Faculty of Engineering, Hiroshima University. From August 1986 through April 1987, he was on leave at Coordinated Science Lab., University of Illinois at Urbana, Urbana, IL, U.S.A., as a visiting associate professor. He joined the Department of Circuits and Systems, Faculty of Engineering, Hiroshima University on July 1, 1990 as an associate professor, and is currently a professor of the Graduate School of Engineering, Hiroshima University. His research interest includes design and analysis of algorithms for graphs, combinatorics, Petri nets, VLSI layout and parallel computation. He is a member of the Institute of Information Processing Society of Japan (IPSJ). 